\title{
Sight-threatening optic neuropathy is associated with paranasal lymphoma
}

This article was published in the following Dove Press journal:

Clinical Ophthalmology

5 March 2010

Number of times this article has been viewed

\author{
Takahiko Hayashi' \\ Ken Watanabe ${ }^{2}$ \\ Yukio Tsuura ${ }^{3}$ \\ Gengo Tsuji ${ }^{4}$ \\ Shingo Koyama ${ }^{4}$ \\ Jun Yoshigi ${ }^{4}$ \\ Naoko Hirata' \\ Shin Yamane' \\ Yasuhito lizima ${ }^{5}$ \\ Shigeo Toyota $^{6}$ \\ Satoshi Takeuchi' \\ 'Department of Ophthalmology, \\ Yokosuka Kyosai Hospital, Japan; \\ 2Department of Hematology, \\ Graduate School of Medicine, Tokyo \\ Medical and Dental University, Japan \\ ${ }^{3}$ Department of Pathology, Yokosuka \\ Kyosai Hospital, Japan; ${ }^{4}$ Department \\ of Radiology, Yokosuka Kyosai \\ Hospital, Japan; ${ }^{5}$ Department of \\ Ophthalmology, Yokohama City \\ University, Japan; ${ }^{6}$ Department of \\ Internal Medicine, Yokosuka Kyosai \\ Hospital, Japan
}

Correspondence: Takahiko Hayashi

Department of Ophthalmology, Yokosuka Kyosai Hospital.I-16,Yonegahamadori,

Yokosuka, Kanagawa, Japan

Email htakahiko-aikou@umin.ac.jp

\begin{abstract}
Malignant lymphoma around the orbit is very rare. We present a rare case of optic neuropathy caused by lymphoma. A 61-year-old Japanese woman was referred to our hospital for evaluation of idiopathic optic neuropathy affecting her right eye. The patient was treated with steroid pulse therapy (methyl-predonisolone $1 \mathrm{~g}$ daily for 3 days) with a presumed diagnosis of idiopathic optic neuritis. After she had been switched to oral steroid therapy, endoscopic sinus surgery had been performed, which revealed diffuse large B cell lymphoma of the ethmoidal sinus. Although R-CHOP therapy was immediately started, prolonged optic nerve compression resulted in irreversible blindness. Accordingly, patients with suspected idiopathic optic neuritis should be carefully assessed when they show a poor response, and imaging of the orbits and brain should always be done for initial diagnosis because they may have compression by a tumor.

Keywords: optic neuropathy, malignant lymphoma, paranasal lymphoma, rhinogenic optic neuropathy
\end{abstract}

\section{Introduction}

Paranasal mass lesions can potentially cause visual loss by compressing the optic nerve, but only a few such cases have been reported in the literature. ${ }^{1-3}$

Here, we present a rare case of optic neuropathy caused by paranasal lymphoma.

This tumor generally has a poor prognosis, and early diagnosis is essential for effective treatment. Imaging modalities such as computed tomography (CT) and magnetic resonance imaging (MRI) are important for making a correct differential diagnosis.

\section{Case report}

A 61-year-old Japanese woman was referred to the Department of Ophthalmology at Yokosuka Kyosai Hospital for evaluation of optic neuropathy affecting her right eye. Her past history included surgery on the paranasal sinuses 50 years earlier, and uterine myomectomy 20 years earlier. There was no relevant family history.

The best corrected visual acuity was counting fingers on the right and 20/30 on the left.

Optic disk and retinal findings were normal. A left relative afferent pupillary defect was present, but the findings on neuro-ophthalmological examination were otherwise unremarkable. There were no abnormal findings on slit-lamp examination, or on general physical examination. The critical flicker frequency was undetectable in the right eye and was $40 \mathrm{~Hz}$ in the left eye. Goldman perimetry showed centrocecal scotoma, indicating an abnormality of the optic nerve. However, fluorescein angiography did 
not reveal any abnormal optic disc findings, such as disc hyperfluorescence or a wedge-shaped filling defect. CT and MRI of the brain and orbits demonstrated a mass occupying much of the ethmoidal sinus (Figure 1A, B). The mass extended into the extraconal right orbit. Since the mass did not seem to be causing compression, the patient was treated with steroid pulse therapy (methyl-predonisolone $1 \mathrm{~g}$ daily for 3 days). This was followed by oral prednisolone, which was started at a dose of $30 \mathrm{mg} /$ day and then tapered. After she was switched to oral steroid therapy, endoscopic sinus surgery was performed under a diagnosis of sinus mucocele by an otolaryngologist. In addition, sinus biopsy was performed.

Despite this treatment, marked improvement of her visual acuity on the right was not obtained and the acuity was $20 / 2000$.

On the day after sinus surgery, the patient developed tender proptosis. The best corrected visual acuity was counting fingers on the right, while ptosis, dilation of the pupil, and loss of eye movements indicated oculomotor nerve palsy. It was impossible to use the Hess chart, because her vision was too poor. MRI showed expansion of the ethmoidal tumor with compression of the right optic nerve and right oculomotor nerve (Figure 1C). On the same day, biopsy revealed diffuse large B cell lymphoma of the ethmoidal sinus (Figure 2A-D). Staging investigations did not show any evidence of disseminated disease, and stage 1E extranodular non-Hodgkin's lymphoma (NHL) was diagnosed. The patient was treated with R-CHOP therapy (rituximab, cyclophosphamide, doxorubicin, vincristine, and prednisolone), followed by loco-regional irradiation at the Hematology Department. The tumor initially responded to treatment and decreased in size. Despite achieving remission, the patient became blind.

\section{Discussion}

Malignant lymphoma around the orbit is very rare, but we should remember that it can threaten vision by compressing the eyeball and/or optic nerve. Malignant lymphoma rarely arises in the paranasal sinuses. For NHL of the head and neck region, less than $5 \%$ of tumors arise at extranodular sites, while the majority develop in the lymphoid tissue of Waldeyer's ring. ${ }^{4}$ Correct diagnosis of paranasal lymphoma is usually delayed. Because tumors at this site cause few early symptoms, the diagnosis is usually made at an advanced stage. Detailed investigation is often postponed at the initial stage and is delayed until the tumor causes clear symptoms. When such a tumor is still small, CT scanning often shows clear paranasal sinuses and leads to a false sense of security.
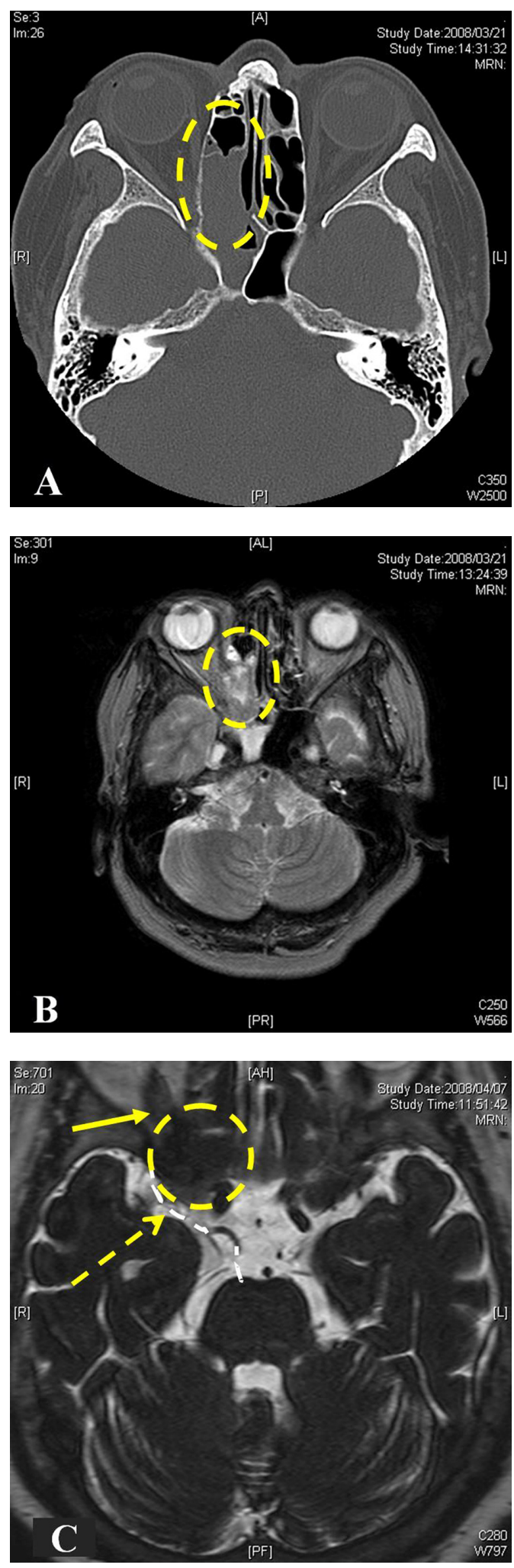

Figure I Tumor mass occupying the frontal sinuses and part of the right anterior ethmoidal sinus. Before steroid pulse therapy, the mass occupies much of the ethmoidal sinus on computed tomography (CT) (A) and magnetic resonance imaging (MRI) (B) T2-weighed MRI showed isointense mass. (C) Despite steroid pulse therapy and endoscopic sinus surgery, the tumor expanded to compress the optic nerve (yellow arrow) and oculomotor nerve. Contrast-enhanced MRI showed low intensity mass in T2 image. (Broken circle indicates the tumor, broken white line indicates the oculomotor nerve and broken yellow arrow indicates the compression point). 

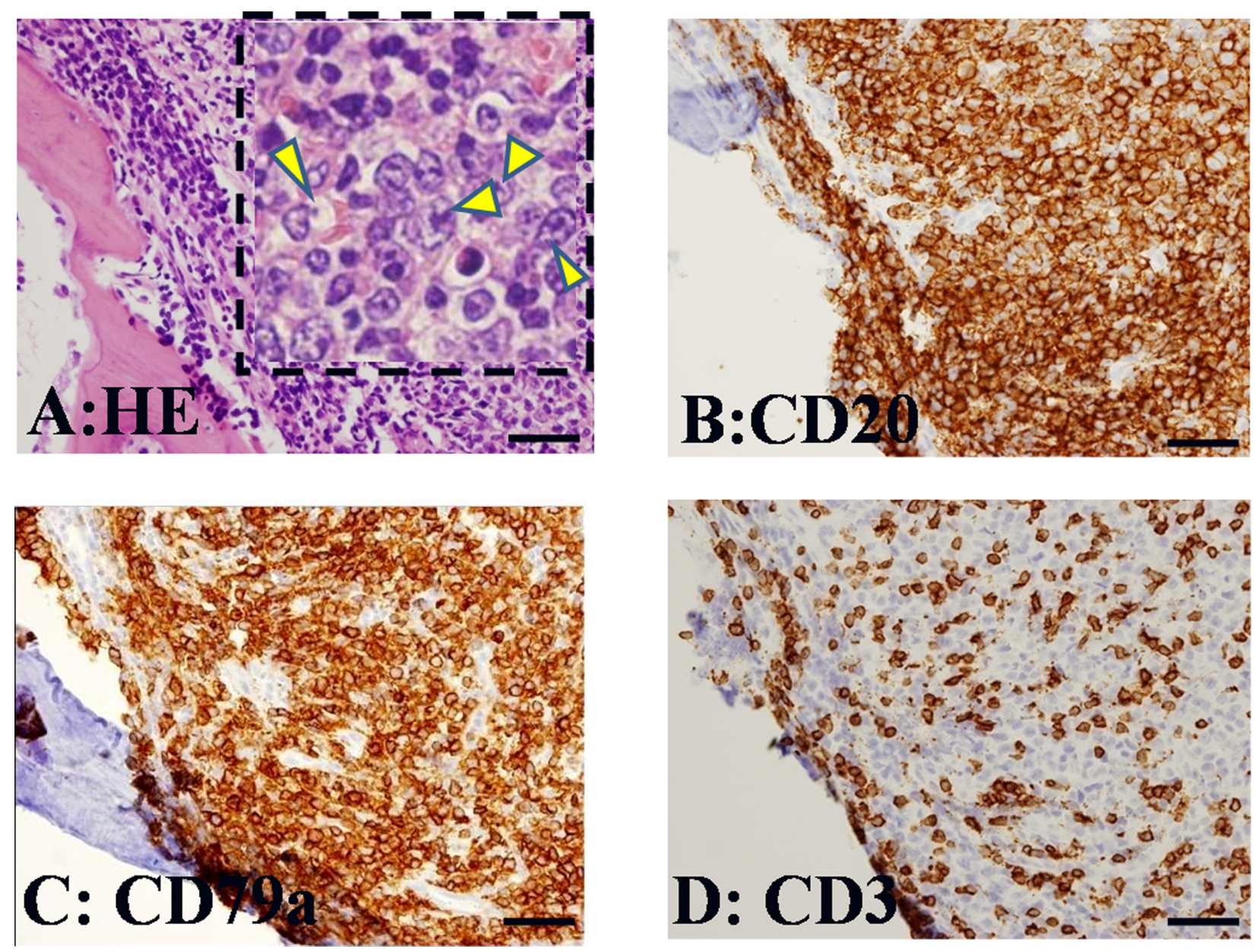

Figure 2 Histological features of the resected specimen. (A) Resected specimen revealed diffuse proliferation of atypical lymphoid cells in the ethmoidal sinus. Insert: There are large-sized cells with prominent nucleoli (arrowheads). These cells express CD20 (B) and CD79a (C), but do not express CD3 (D). These immunohistochemical reactions are consistent with that of diffuse large B cell lymphoma.

However, sometimes obstruction of a sinus will create a mucocele or pyocele, which is detected by surgery. ${ }^{5}$ Orbital invasion is a common manifestation of malignant sinus tumors, and ocular findings can be useful to assess the expansion and status of a primary sinus lesion when clinical evaluation is difficult. The common presenting signs and symptoms are proptosis, visual loss, lid edema, diplopia, limitation of ocular motility, conjunctival chemosis, blepharoptosis, conjunctival injection, globe displacement, epiphora, anisocoria, eye pain, and photophobia. ${ }^{6}$ From $50 \%$ to $70 \%$ of the patients with orbital and ocular manifestations show orbital involvement at the time of initial radiological evaluation. ${ }^{6,7}$ The abnormalities detected on X-ray films of the sinuses range from a soft tissue mass to orbital bone destruction. The most common sinus to be involved by malignancy is the maxillary sinus and squamous cell carcinoma is the most frequent malignant tumor. ${ }^{6}$

The available reviews include only a few cases of sinonasal lymphoma. The majority of sinonasal lymphomas are classified as clinical stage 1E by the Ann Arbor system, and as large B cell lymphoma by the REAL classification. ${ }^{8,9}$

Some studies have suggested that radiation alone provides good regional control at an early stage, while additional chemotherapy can be reserved for more extensive disease. ${ }^{10,11}$ However, other studies have shown that combined modality treatment with chemotherapy and loco-regional irradiation improves both disease-free and overall survival. ${ }^{12}$ The overall mortality rate is $55 \%{ }^{8,9,13}$ For effective treatment of these lifethreatening malignant tumors, early detection by investigation of orbital symptoms may be important.

Our initial diagnosis was idiopathic optic neuritis because we did not consider optic nerve compression based on the imaging findings. However, repeat imaging after the diagnosis had been made revealed evidence of compression, suggesting that the patient already had optic nerve compression which the initial imaging study failed to detect. We started R-CHOP therapy immediately after we identified malignant lymphoma, 
but prolonged optic nerve compression resulted in irreversible blindness. Despite this, we do not think that starting therapy earlier would have led to a good outcome because vision was already reduced to counting fingers (indicating irreversible damage) at her initial presentation. Accordingly, patients with suspected idiopathic optic neuritis should be carefully assessed when they show a poor response, and imaging of the orbits and brain should always be performed for initial diagnosis because they may have compression by a tumor. This case serves to remind ophthalmologists about the pitfalls of diagnosing and treating optic neuritis. If ophthalmologists investigate patients who have headache or a history of chronic sinusitis with bone destruction on X-ray films, they should strongly suspect a malignant tumor of the sinuses.

\section{Disclosures}

The authors disclose no conflicts of interest.

\section{References}

1. Johnson LN, Hepler RS, Yee RD, et al. Sphenoid sinus mucocele (anterior clinoid variant) mimicking diabetic ophthalmoplegia and retrobulbar neuritis. Am J Ophthalmol. 1986;102:111-115.

2. Dunya IM, Frangieh GT, Heilman CB, et al. Anterior clinoid mucocele masquerading as retrobulbar neuritis. Ophthal Plast Reconstr Surg. 1996;12:171-173.
3. Chou PI, Chang YS, Feldon SE, et al. Optic canal mucocele from anterior clinoid pneumatisation. Br J Ophthalmol. 1999;83:1306-1307.

4. McNelis FL, Pai VT. Malignant lymphoma of the head and neck. Laryngoscope. 1969;79:1076-1087.

5. Robbins KT, Fuller LM, Vlasak M, et al. Primary lymphomas of the nasal cavity and paranasal sinuses. Cancer. 1985;56:814-819.

6. Johnson LN, Krohel GB, et al. Sinus tumors invading the orbit. Ophthalmology. 1984;91:209-217.

7. Nemet AY, Deckel Y, Kourt G. Orbital invasion of frontal sinus lymphoma. Orbit. 2006;25:149-151.

8. Hatta C, Ogasawara H, Okita J, et al. Non-Hodgkin's malignant lymphoma of the sinonasal tract- treatment outcome for 53 patients according to REAL classification. Auris Nasus Larynx. 2001;28:55-60.

9. Frierson HF, Mills SE, Innes DJ. Non-Hodgkin's lymphomas of the sinonasal region: histologic subtypes and their clinicopathologic features. Am J Clin Pathol. 1984;81:721-727.

10. Shikama N, Izuno I, Oguchi M, et al. Clinical stage IE primary lymphoma of the nasal cavity: radiation therapy and chemotherapy. Radiology. 1997;204:467-470.

11. Proulx GM, Caudra-Garcia I, Ferry J, et al. Lymphoma of the nasal cavity and paranasal sinuses: treatment and outcome of early stage disease. Am J Clin Oncol. 2003;26:6-11.

12. Hausdorff J, Davis E, Long G, et al. Non-Hodgkin's lymphoma of the paranasal sinuses: clinical and pathological features, and response to combined-modality therapy. Cancer J Sci Am. 1997;3:303-311.

13. Logsdon MD, Ha CS, Kavadi VS, et al. Lymphoma of the nasal cavity and paranasal sinuses: improved outcome and altered prognostic factors with combined modality therapy. Cancer. 1997;80:477-488.
Clinical Ophthalmology

\section{Publish your work in this journal}

Clinical Ophthalmology is an international, peer-reviewed journal covering all subspecialties within ophthalmology. Key topics include: Optometry; Visual science; Pharmacology and drug therapy in eye diseases; Basic Sciences; Primary and Secondary eye care; Patient Safety and Quality of Care Improvements. This journal is indexed on

\section{Dovepress}

PubMed Central and CAS, and is the official journal of The Society of Clinical Ophthalmology (SCO). The manuscript management system is completely online and includes a very quick and fair peer-review system, which is all easy to use. Visit http://www.dovepress.com/ testimonials.php to read real quotes from published authors. 